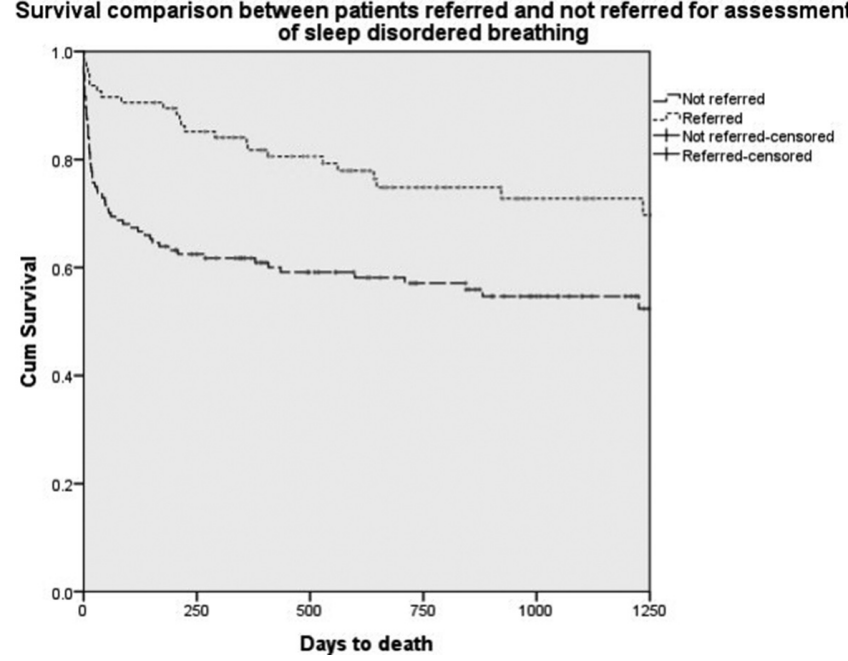

Abstract P110 Figure 1

Conclusions Rates of obesity and persistent hypercapnia are high in survivors of critical illness. However, patients are frequently not referred for specialist respiratory assessment. Survival is increased in patients referred for long-term management, although this data needs to be interpreted with caution as this could be the result of referral bias and a prospective study is now required.

\section{P111 RESPIRATORY FLOW LIMITATION IN THE ABSENCE OF OBSTRUCTIVE SLEEP APNOEA RESPONDS TO CPAP THERAPY}

B Chakrabarti, S Emegbo, S Craig, I Heseltine, T Wright, N Duffy, JF O'Reilly. University Hospital Aintree, Liverpool, UK

\subsection{6/thoraxjnl-2015-207770.248}

Background The Apnoea-Hypopnoea index (AHI) is regarded as a gold standard diagnostic marker of Obstructive Sleep Apnoea Syndrome (OSAS). However, a number of patients present with excessive daytime sleepiness (EDS), yet exhibit a raised Respiratory Disturbance Index (RDI), comprising of flow limited breaths in the presence of a normal AHI $(<5$ events/hr). We sought to evaluate the benefits of CPAP in this "Flow Limitation" cohort compared to a matched population of OSAS subjects.

Results 27 subjects (Mean age 47 (SD 8) years; ESS 17(4); 48\% male; BMI 35.60 (8.12)) presented to our Sleep Service with EDS and undertook a cardio-respiratory polysomnograph, demonstrating an RDI $>15$ and AHI $<5$ (Mean RDI 16 (4); AHI 3 (2); ODI 5(3)) 25 subjects were subsequently treated with CPAP. At "6-week compliance" visits, $20(80 \%)$ were deemed compliant with CPAP (mean nightly usage 6.03 (1.47) hrs; pre-CPAP ESS 18(3) falling to 9 (4) following CPAP. Within the Flow Limitation cohort, statistically significant associations were observed between CPAP compliance and Female gender (100 v 55\%), higher BMI (36.61 v 31.56), higher pre-CPAP ESS (18 v13) and lower Pulse Transit Time PTT (300.90 v 316 ms).

This "Flow Limitation" cohort was compared with an age/ gender matched "OSAS "cohort (ESS 15(5); BMI 38.33 (7.80) AHI 58.16 (25.79) ODI 48 (23)). 26 OSAS subjects were treated with CPAP with 19 (70\%) deemed compliant (nightly usage 5.25 (3.55) hrs; pre-CPAP ESS 16 (5) falling to 10(5) following CPAP. Whilst the mean PTT of the OSAS cohort was lower than the
"Flow Limitation" cohort, this did not reach statistical significance $(298.85(15.04)$ v 306.44 (18.36) ms; ANOVA; $\mathrm{p}=0.1$ ) yet the PTT Deceleration Index (DI), a surrogate of physiological arousal, was significantly higher in the OSAS cohort (59.05 (29.33) v 36.32(23.69)/hour; ANOVA; $\mathrm{p}=0.003)$.

Conclusion "Sleepy" subjects exhibiting an elevated Flow Limitation Index in the presence of a normal AHI appear to demonstrate a response to CPAP therapy comparable to that observed in OSAS. Female gender and a higher BMI appear to predict compliance with therapy, whilst the utility of Pulse Transit Time in guiding decision making in "sleepy" subjects with a normal AHI merits further study.

\section{P112 CPAP ROLE ON THE PERIOPERATIVE OUTCOMES OF PATIENTS WITH OBSTRUCTIVE SLEEP APNOEA}

VM Macavei, D King, J Sumpter, M Berger, OE Mohr, J Mitic, TC O'Shaughnessy. Newham University Hospital, Barts Health NHS Trust, London, UK

\subsection{6/thoraxjnl-2015-207770.249}

Background Obstructive sleep apnoea (OSA) has been previously reported as an independent risk factor for intra and post operative adverse events. ${ }^{1}$

Early diagnosis of sleep disordered breathing and initiation of CPAP treatment was suggestive to improve operative outcomes. ${ }^{2}$ Objectives To determine the prevalence of sleep apnoea in a surgical population and establish the role of CPAP on peri-operative outcomes in patients with OSA.

Methods A retrospective study was performed in a university hospital between $1^{\text {st }}$ June 2013 and $1^{\text {st }}$ June 2015 and included 160 surgical patients investigated for OSA. Sleep apnoea was defined as dip rate $>10$ events/hour associated with a desaturation of $4 \%$ below the baseline. Statistical analysis was performed with STATA v10 software.

Results From 160 surgical patients included, 33.1\% (53) were females and average age was 54 years. Prevalence of OSA was $44.3 \%(71 / 160)$ and $12.5 \%(20 / 160)$ had severe OSA defined as a dip rate $>30$ events/hour.

Following sleep investigations, 68 patients had surgical interventions: $48.5 \%(33 / 68)$ trauma and orthopaedics, $17.6 \%(12 /$ $68)$ general surgery, $10.2 \%$ (7/68) urology, $8.8 \%(6 / 68)$ gynaecology, 7.3\% (5/68) colorectal, 4.4\% (3/68) ENT. From 68 patients undergoing surgical procedures, 44.1\% (30/68) were diagnosed with OSA and started on CPAP prior to surgery.

Peri-operative adverse events were not significantly related to OSA when compared to non OSA patients: intra operative desaturations $(23.3 \%$ vs $26.3 \%)$ and prolonged recovery stay $(53.3 \%$ vs $55.2 \%)$.

OSA patients had a lower hospital stay compared to non OSA group (1.7 vs 3.1 days).

Conclusions We have identified a high prevalence of sleep apnoea of $44 \%$ in surgical population. CPAP treatment was found effective in improving operative outcomes of patients with OSA, further studies being needed to confirm these results. Routine pre-assessment screening for OSA followed by sleep investigations for initiation of CPAP prior to surgery is recommended.

\section{REFERENCES}

1 Vasu TS, Grewal R, Doghramii K. Obstructive sleep apnoea syndrome and perioperative complications. J Clin Sleep Med. 2012;8(2):199-207

2 Gross JB, et al. Practice guidelines for the perioperative management of obstructive sleep apnea: ASA task force on perioperative management of OSA. Anesthesiology 2014;120 